\title{
BEM analysis of rc cracked bridge slabs
}

\author{
M.Y. Minch, A. Kmita
}

\begin{abstract}
The purpose of this work is to present some aspects and main problems of theory and computations of RC (reinforced concrete) bridge slabs. The differential equation of the cracked bridge slab, using the classical variational method is worked out. The equation of bridge slab deflection with the boundary conditions and compatibility conditions in the crack are obtained. The total differential equations in the class of the general functions are shown. In this model the effect of discontinuity of the first derivative of deflection functions is taken. The numerical results of an approximate method of solutions of RC cracked plates with boundary element method (BEM) are shown.
\end{abstract}

Keywords - Boundary Element Method (BEM), analysis, reinforced concrete (rc), crack, slab, bridge

\section{Introduction}

Reinforced concrete constructions exhibit very complicated behavior that widely differs from homogenous, i.e. steel structure. The structural reinforced concrete (RC) system is composed of different materials, such as cement, steel bars, aggregate, etc. Moreover each material shows various physical phenomena. These heterogeneities and additional defects that are caused by cracks in the concrete, lead to the non-linear behavior of entire structure. If the defects that are caused by the cracks in the concrete are treated as continuous functions, this gives an unsatisfied solution because of the summation of the assumption and solution errors. Therefore adequate mathematical modeling of the construction is so important since the final error appears solely in solution phase.

The real behaviour of reinforced concrete bridge slabs differs widely from the results of a linear elastic computation. At the beginning of loading RC construction shows non-linear deformations because of non-linear concrete stress-strain relation. As an example of such behaviour, the characteristic load-midpoint deflection curve $(P-w)$ of RC rectangular slab shown by Borcz (1963) is demonstrated in Fig.1. Even under working loads $\boldsymbol{P}_{u}$ and obviously up to failure loads $\boldsymbol{P}_{g r}{ }^{I I I}$, the curve is extremely non-linear that is additionally caused by non-linear stressstrain relation of reinforcement bars and discontinuity of the slab.

The first change of the stiffness is caused by the beginning of cracking for cracking load $\boldsymbol{P}_{r y s}$. Construction becomes heterogeneous as a result of cracks and bond-slip between concrete and reinforcement. The crack, treated as a

\section{Maciej Yan Minch}

Wrocław University of Technology

Poland

\section{Andrzej Kmita}

Wrocław University of Technology Poland defect, causes the zoning of the structure region. Each of the zones is connected with another one by means of reinforcement bars appearing in the cracks. So, the edges of the cracks are not free from tension at the points of connections and simultaneously the general vector of displacements has a jump equal to the opening of the crack. Additionally, the forces of the broken adhesion between steel bars and concrete as well as some other transverse forces appear in crack areas. Under greater loading the further propagation of cracks are shown up to second reduction of the stiffness, when the reinforcement begins to yield $\boldsymbol{P}_{g r}^{I I}$. This finally leads to the failure load $\boldsymbol{P}_{g r}{ }^{I I I}$ associated with the crushing of concrete. For load $\boldsymbol{P}_{g r}{ }^{I V}$, the slab reaches limiting membrane state. The forces as discussed above, can cause a fundamental redistribution of stresses in the cracked construction compared with the homogeneous one. This thesis has been proved by a number of experimental tests for RC slabs.

As a result of stiffness reduction caused by cracking, the values of bending moments are lower compared with those ones obtained from the linear elastic theory. Thus the reinforcement in cracked zones could be divided in a better possible manner. Additionally as a result of bending moments that are decreasing in cracked zones, RC slab can be more loaded before reaching limited strain (point E) or local failures (point F).

A typical characteristic disloading and reloading line has been shown in Fig. 1. The separation of elastic $w^{e}$ and plastic deflection $w^{p}$, has been assumed according to small strain theory. Such assumption makes computation for this process much easier.

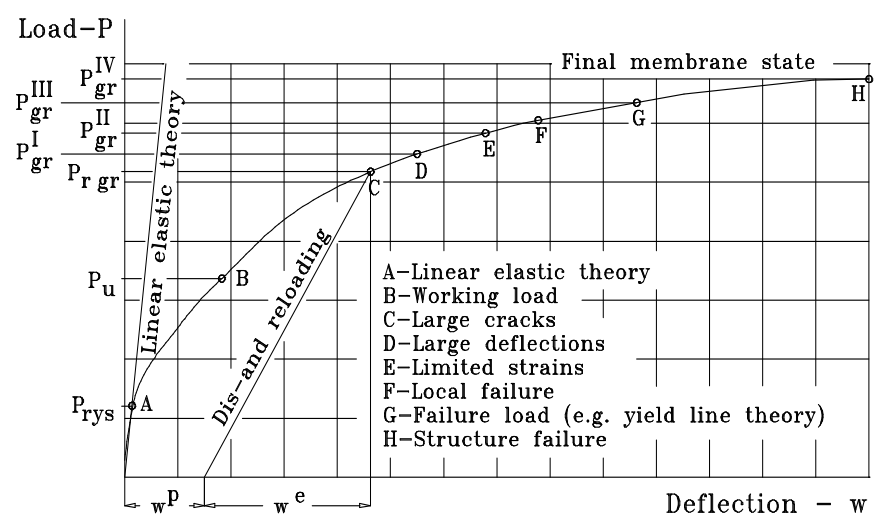

Figure 1. Characteristic load-midpoint deflection curve of RC slab.

The purpose of this paper is to present some aspects and main problems of distribution theory and computations of $\mathrm{RC}$ bridge slabs. The first investigations for distribution model of RC beam and slabs with cracks were made by Borcz [1]. This paper expands distribution beam and slab Borcz's models for RC cracked bridge slabs. It was assumed that computations are valid for service loads because most of the constructions are loaded within the limits (that is, limits between points $\mathrm{A}$ and $\mathrm{C}$ ). In this loading area the linear theory of elasticity as well as yield line theory appears to be not valid. 
This paper contains a mathematical model of a reinforced concrete planar structure formulated in terms of general functions of distribution theory of Schwartz [2]. The physical hypothesis about discontinuous change of displacement, caused by the cracking of the extension zone in the concrete, with the associated fundamental solution and the assumption of rotating cracks, is included in the model. Stress intensity factors can be calculated using Jintegral or other methods. The mathematical model is formulated for two-dimensional space that can solve problems in elastostatics, plate bending or Poisson's equations. The model of bending bridge slabs includes the horizontal forces what cause conjugation of the plane stress state and the bending state and additionally allow to calculate the prestressed construction. The boundary element method has proven to be an effective technique for calculating stresses, displacements and stress intensity factors in crack analysis. Here the application of non-linear boundary element method (BEM) is used to the analysis of cracked RC bridge slabs. The results of numerical examples are presented below.

\section{Assumption}

The arbitrary slab as shown in Fig. 2 is considered. The slab has arbitrary homogeneous boundary conditions and is arbitrary forced with load $p$.

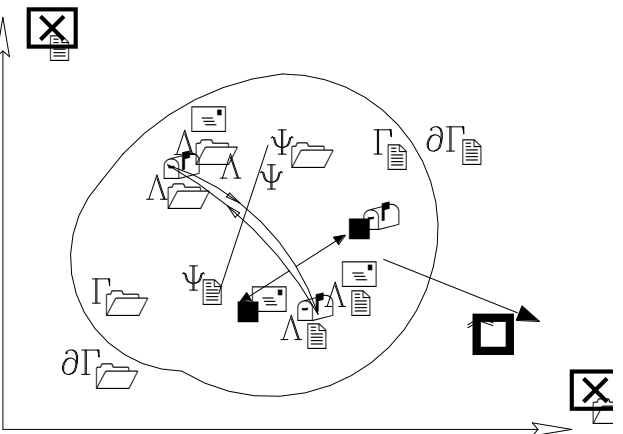

Figure 2. Scheme of the slab $\Gamma$ with $\operatorname{crack} \Lambda$.

The region of slab $\Gamma$ is divided by the curve $\Lambda$, means the crack, in two zones $\Gamma_{1}$ and $\Gamma_{2}$ with bound $\partial \Gamma_{1}$ and $\partial \Gamma_{2}$. The curve $\Lambda$ has two ends $\Lambda_{1}$ and $\Lambda_{2}$. The normal external direction cosines $\mathbf{n}$ of the edge $\Lambda$ of regions $\Gamma_{l}$ and $\Gamma_{2}$ have different sign. The crack line has been crossed by the line $\Psi$, means the reinforcement bar. After cracking the real steel bar appears inside the crack area and connects the both sides of the crack. The line $\Psi$ outside the crack area symbolize the line of broken adhesion where the bond-slip occurs. The considered model can be easy generalized to any amount of cracks $\Lambda$ and steel bars $\Psi$.

\section{Material properties}

The concrete properties should be included in the biaxial domain. Therefore the biaxial stress-strain relation and the failure criterion of concrete depends on the results of the tests that are performed to obtain these relations. The biaxial tests of Kupfer [3] for short time loading and proportionally increasing load proved to be the most reliable. Link [4] developed an incremental formulation for the tangent stiffness of the concrete on the basis of Kupfer's tests (see
Fig.3). The stresses are normalized in terms of the uniaxial cylinder strength, in order that the formulation can be used for different grades of concrete. The failure criterion cannot be used as plasticity condition, because it describes a boundary for the maximum stresses and does not allow any statements about the plastic deformations.

The envelope of the limit curve is described by the three subdomains. First subdomain of biaxial tension (I) has the following limit stress in concrete:

$$
\sigma_{1}=\sigma_{2}=\mathrm{f}_{\mathrm{ct}} .
$$

Second subdomain (II) for region of tension-compression can be written as follows:

$$
\begin{aligned}
& \frac{\sigma_{1}}{f_{c t}}=1+0,8 \frac{\sigma_{2}}{f_{c}} \text { for } \sigma_{2} \geq \approx-0,95 f_{c}, \\
& \frac{\sigma_{2}}{f_{c t}}=1+0,8 \frac{\sigma_{1}}{f_{c}} \text { for } \sigma_{1} \geq \approx-0,95 f_{c} .
\end{aligned}
$$

Third subdomain (III) for region of biaxial compression can be written with following formulas:

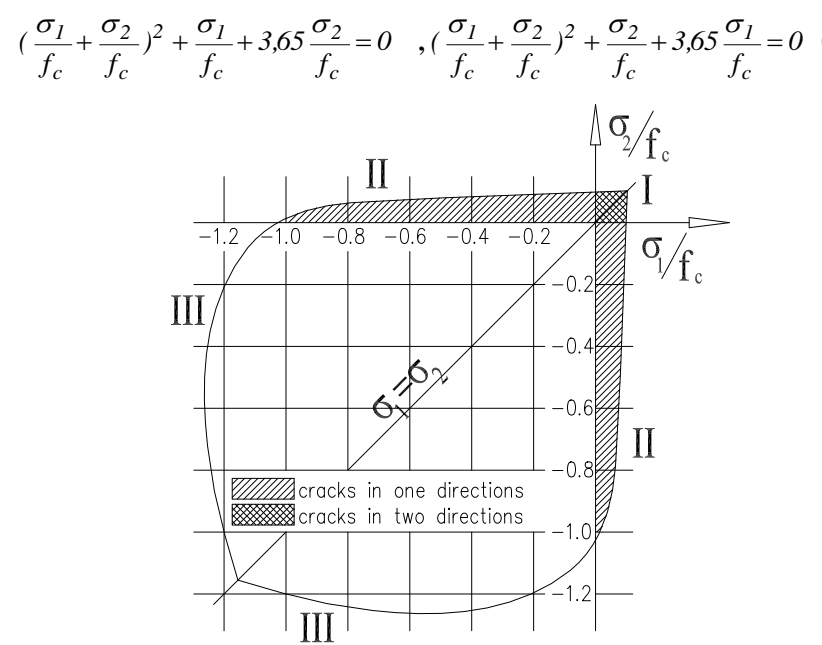

Figure 3. Biaxial state of stresses by the formulation of Link [4]

The comparison of stress-strain relation between test results of Kupfer [3] and analytical formulation of Link [4] was shown on Fig. 4. The demonstrated above concrete physical law of Link was used in the computation of the planar structure within the presented method.

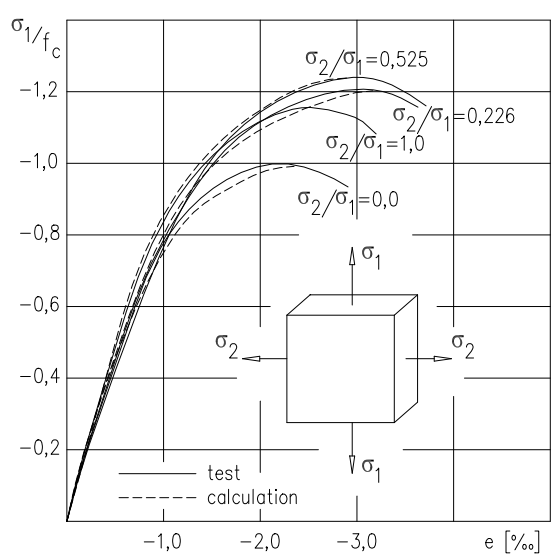

Figure 4. Stress-strain relation of concrete.

The stress-strain relation of steel bars was taken as a well-known elastoplastic relation from uniaxial tests. 


\section{Iv. Bond-Slip characteristic}

After the cracking of the concrete, the tensile forces in the cracked area are transmitted by bond to the reinforcement that consists of steel bars. Along the segments of broken adhesion the steel bar cooperates with the concrete through the tangential stresses distributed on the perimeter of the bar. The slip is defined as a relative displacement between reinforcement bars and surrounding concrete. The increment of tensile stresses in the steel bar was approximated by the third-degree curve. Hence, the tangential stresses and bond-slip relationships, as representation of the stiffness of the bond has been found to be in agreement with the tests of Dörr \& Mehlhorn [5], (i.e., the second-degree distribution along the segment $l_{f}$, where $l_{f}$ means distance between cracks).

Some characteristic bond-slip relations of the tests of Dörr \& Mehlhorn [5] under different transverse compression are shown in Fig. 5.

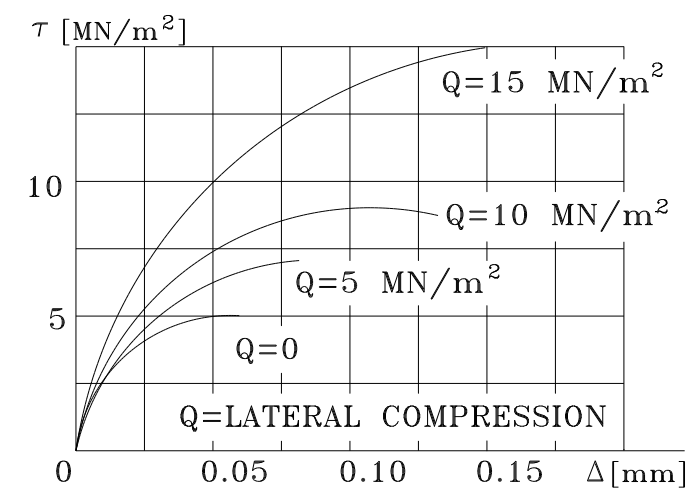

Figure 5. Comparison of the $\tau-\Delta$ relationship with lateral pressure.

\section{v. Creep of concrete}

The creep function presented by Bažant \& Panula [6] (for basic and drying creep and shrinkage) is most suitable for concrete structures because the parameters can be calculated only from the concrete composition. What more, the values obtained from his formulae agree with experimental data very well. For calculation, the model developed by Bažant \& Panula [6] was used.

The creep function for drying was written as follows:

$$
J^{\prime}(t, \tau)=J(t, \tau)+C_{d}\left(t, \tau, t_{0}\right)-C_{p}\left(t, \tau, t_{0}\right),
$$

where $J(t, \tau)$ - basic creep; $C_{d}$ - increase of creep caused by drying; $C_{p}$ - decrease of creep after drying (with beginning time of drying $\left.-t_{0}\right)$.

Particularly, for calculation the approximate relaxation function is very useful:

Particularly, for calculation the approximate relaxation function is very useful:

$$
R(t, \tau)=\frac{0.992}{J(t, \tau)}-\frac{0.115}{J(t, t-1)}\left[\frac{J(\tau+\xi, \tau)}{J(t, t-\xi)}-1\right]
$$

Thanks to that, the value of $E^{\prime \prime}(t, \tau)$ can be written as follows:

$$
E^{\prime \prime}(t, \tau)=\frac{1-J(t+1, \tau) R(t, \tau)}{J(t, \tau)-J(t+1, \tau)}
$$

and the relationship between stress and strain in concrete has the following form:

$$
\sigma_{c}(t)=E^{\prime \prime}\left(t, t_{0}\right) \alpha(t)+A,
$$

where $A=\sigma_{c}\left(t_{0}\right)\left[1-E^{\prime \prime}\left(t, t_{0}\right) J\left(t, t_{0}\right) ; \alpha(t)\right.$ - strain in pressed side of a slab.

The creep function of concrete was taken from the rheological model with the creep function as below ( $f_{c}{ }^{\prime}$ means the 28 days compressive strength of concrete):

$$
J(t, \tau)=\frac{1}{E_{o}\left(f_{c}^{\prime}\right)}\left[1+\varphi_{1}\left(f_{c}^{\prime}\right)\left(\tau^{-m\left(f_{c}^{\prime}\right)}+\alpha\right)(t-\tau)^{n\left(f_{c}^{\prime}\right)}\right] .
$$

The presupposition similar to the linear creep theory of concrete in compression is used for bond creep with bond creep coefficient $\varphi_{b}$. Naturally in accordance to $\tau$ - $\Delta$ relationship of Dörr \& Mehlhorn [5], bond creep cannot be described by linear theory. The model of Rotasy \& Keep [7] was applied to describe the creep of the bond in the cracked concrete, where the time-dependence of bond in the loaded state exhibits a similar behavior as concrete in compression.

\section{vI. Constitutive laws for crack width}

The physical law of the crack opening $\mathbf{r}$ was taken from the beam analogy formulated by Borcz [1], the general assumption of the cracks' theory and the equilibrium conditions in the crack. This can be written in the following incremental form:

$$
\mathbf{r}(s)=\mathbf{r}^{p}(s)+\mathbf{r}^{e}(s) \Im\left(\dot{\mathbf{N}}_{n}, \dot{\mathbf{M}}_{n}\right)
$$

where $\mathbf{N}_{\boldsymbol{n}}$ - tensile vector; $\mathbf{M}_{\boldsymbol{n}}$ - bending moment normal to the crack line $s ; \mathfrak{I}$ - nonlinear function of $\mathbf{N}$ and $\mathbf{M}$.

The zone of slab $\Gamma_{1}$ is connected with another one $\Gamma_{2}$ by means of reinforcement bars appearing in the cracks. So, the edges of the cracks are not free from tensions at the points of connections. The components $\mathbf{r}$ of (9) are given from RC element tests as well as from general assumption of crack theory and equilibrium conditions in the crack.

Note, that the vector $\mathbf{r}$ (i.e., modified Burgers vector depending additionally on inner forces in the crack) is divided on elastic $\mathbf{r}^{e}$ and plastic $\mathbf{r}^{p}$ parts respectively. Such formulated modeling allows to assume any arbitrary form of the physical laws of the crack's width.

\section{vII. Mathematical model of the bridge slab}

It was assumed that the crack $s$ exists in the region of the structure $\Gamma$ with the edge $\Gamma_{B}=\partial \Gamma$ and crack $\Lambda=\Gamma_{c}$. The crack is connected with the local coordinate system $(s, n)$.

The discontinuous variational problem of surface integral is considered. The difference of internal and external forces works $U_{\Gamma}$ and $\mathbf{p}(\mathbf{x}) w$ respectively, taken from linear theory of elasticity as well known relation moment-deflection-curvature $\mathbf{M}=\mathbf{M}(\kappa(w))$ has the form: 


$$
J[w(w(\mathbf{x}))]=\int_{\Gamma}\left[U_{\Gamma}(D \mathbf{M} f(w(\mathbf{x})))-\mathbf{p}(\mathbf{x}) w(\mathbf{x})\right] d \Gamma
$$

where $D$ - stiffness of the cracked plate; $\mathbf{M}$ - vector of moments; $\kappa(\mathbf{x})$ - curvature as a second derivative of deflection $w$.

We are looking for the extreme of the functional (10) with set of permissible values of deflection $w\left(x_{1}, x_{2}\right)$ in the region $\Gamma$. As we assumed above the crack $\Lambda$ divides region $\Gamma$ into $\Gamma_{1}$ and $\Gamma_{2}$ respectively.

The searching function $w(\mathbf{x})$ is in the class of function $w$ $\in \mathrm{C}^{0}(\Omega) \cap \mathrm{C}^{4}(\Omega / \Lambda) \quad$ (for $\mathbf{x} \in \Lambda$ function $w^{\prime}(\mathbf{x})$ has singularity).

Necessary condition for $w$ as a real deflection of slab is zeroing of first variation of functional (10). By applying Green's integration by parts we can change the surface integral as a sum of surface and curvilinear integral respectively. With moments $\mathbf{M}$ as a function of curvatures $\kappa \Rightarrow \mathbf{M}=\mathbf{M}(\kappa(w))$ we obtain differential equation of the slab:

$$
\Delta \Delta w(\mathbf{x})=\frac{1}{D} \mathbf{p}(\mathbf{x}), \quad \mathbf{x} \in \Gamma_{1} \cup \Gamma_{2},
$$

associated with combination of elementary boundary conditions:

$$
\left.\widetilde{\nabla}(w)\right|_{\partial \Gamma}=\left.O \vee w\right|_{\partial \Gamma}=0,\left.\quad \tilde{\mathbf{M}}(w)\right|_{\partial \Gamma}=\left.O \vee \frac{\partial w}{\partial n}\right|_{\partial \Gamma}=0,
$$

and compatibility condition in the crack:

$$
\left.\widetilde{\nabla}(w)\right|_{\Lambda}=\left.0 \vee w\right|_{\Lambda}=0,\left.\quad \tilde{\mathbf{M}}(w)\right|_{\Lambda}=\left.0 \vee \frac{\partial w}{\partial n}\right|_{\Lambda}=0
$$

Here $\tilde{\mathbf{V}}(w), \tilde{\mathbf{M}}(w)$ are differential operators as follows:

$$
\tilde{\mathbf{M}}=v \Delta+(1-v) \frac{\partial^{2}}{\partial n^{2}}, \quad \tilde{\nabla}=\frac{\partial \Delta}{\partial n}+(1-v) \frac{\partial}{\partial s}\left[\frac{\partial}{\partial s}\left(\frac{\partial}{\partial n}\right)-\kappa \frac{\partial}{\partial s}\right],
$$

where $\kappa$-curvatures in fixed point on the crack line $\Lambda$.

Here $[.]_{\Lambda}$ means difference of left and right side limit of expression in square braces on the curve $\Lambda$ :

$$
[f]_{s}=\lim ^{+} f-\lim ^{-} f .
$$

It can be easily shown that using the assumption of the distribution theory of Schwartz [2], constitutive law of defect (9) and properties (12)-(14), the eq. (11) has the following form:

$$
\begin{gathered}
\langle\Delta \Delta w(\mathbf{x}), \varphi\rangle=\left\langle\frac{1}{D} \mathbf{p}(\mathbf{x}), \varphi\right\rangle+\int_{\partial \Gamma}\left[w \widetilde{\nabla}(\varphi)+\widetilde{\mathbf{M}}(w) \frac{\partial \varphi}{\partial n}-\widetilde{\nabla}(w) \varphi-\right. \\
\left.\frac{\partial w}{\partial n} \tilde{\mathbf{M}}(\varphi)\right] d \Gamma+\int_{\Lambda} \mathbf{r}(\mathbf{x}) \widetilde{\mathbf{M}}(\mathbf{x}) d \Lambda+(v-1) \varphi \frac{\partial r}{\partial s} \mid \begin{array}{l}
\Lambda_{2} \\
\Lambda_{1}
\end{array} .
\end{gathered}
$$

The differential equation of deflection $w(\mathbf{x})$ in terms of general functions was obtained.

Using functional way of description with distribution in form of Dirac's- $\delta$ we can write final general differential equation of cracked slab, appropriate boundary and compatibility conditions in the crack respectively:

$$
\begin{gathered}
\Delta \Delta w=\frac{1}{D} \mathbf{p}(\mathbf{x})+\tilde{\mathbf{M}}\left(\mathbf{r}(\mathbf{x}) \delta_{\Lambda}\right)-\left[\frac{\partial \Delta}{\partial n}+(1-v) \frac{\partial}{\partial s}\left(\frac{\partial^{2}}{\partial \partial \partial}+\kappa \frac{\partial}{\partial s}\right)\right]\left(w \delta_{\partial \Omega}\right)- \\
\frac{\partial}{\partial n}\left(\tilde{\mathbf{M}}(w) \delta_{\partial \Omega}\right)-\tilde{\mathbf{M}}\left(\frac{\partial w}{\partial n} \delta_{\partial \Omega}\right)-\tilde{\mathbf{V}}(w) \delta_{\partial \Omega} .
\end{gathered}
$$

\section{vIII. Mathematical model of the plane stress plate}

As was assumed above the plate can be also loaded in its plane. It means that the problems of presstresed slabs can be solved. The horizontal loads may act on the edge for presstresing model of the slab with Jacks as well as inside the slab on the line $\Psi$ where pre-tensioning of the slab is realized.

The discontinuous variational problem of surface integral for displacement (plane stress state), in the same way as considered above for bending slab, was solved. The equilibrium equations, constitutive law and strain equations are assumed to be represented by well-known theory of elasticity relations. Analogue to equation 10 we are looking for the extreme of the functional of strain energy $U_{\Gamma}$ with set of permissible displacement value $\mathbf{u}(\mathbf{x})$ :

$$
J[\mathbf{u}(\mathbf{x})]=\int_{\Gamma} U_{s}(\mathbf{u}(\mathbf{x})) d \Gamma-\int_{\Gamma} \mathbf{b}(\mathbf{x}) \mathbf{u}(\mathbf{x}) d \Gamma-\int_{\partial \Gamma} \mathbf{p}(\mathbf{x}) \mathbf{u}(\mathbf{x}) d \partial \Gamma,
$$

where $\mathbf{u}(\mathbf{x})$ - displacement vector; $\mathbf{b}(\mathbf{x})$ - body forces.

The searching function $\mathbf{u}(\mathbf{x})$ is in the class of function $\mathbf{u} \in \mathrm{C}^{2}(\Omega / \Lambda)$ (for $\mathbf{x} \in \Lambda$ function $\mathbf{u}(\mathbf{x})$ has singularity).

Applying Green's transformation with relations stressstrain-displacement S-E-u we obtain differential equation of plane stress plate:

$$
\mu\left(\nabla^{2}+\frac{3 \lambda+2 \mu}{\lambda+2 \mu} \operatorname{grad} \operatorname{div}\right) \mathbf{u}(\mathbf{x})+\mathbf{b}(\mathbf{x})=0,
$$

associated with combination of elementary boundary conditions:

$$
\widetilde{\mathbf{P}}(\mathbf{u}(\mathbf{x}))=\mathbf{p}(\mathbf{x}), \quad \text { for } \mathbf{x} \in \Gamma_{1} \cup \Gamma_{2},
$$

and compatibility condition in the crack:

$$
[\widetilde{\mathbf{P}}(\mathbf{u}(\mathbf{x}))]_{\Lambda}=0, \quad \text { for } \mathbf{x} \in \Lambda_{1} \Lambda_{2},
$$

where $\lambda, \mu$-Lame' constants; $\widetilde{\mathbf{P}}$ - operator of surface tension:

$$
\widetilde{\mathbf{P}}(.)=\mu\left(\tilde{\nabla}+\frac{2 \lambda}{\lambda+2 \mu} \mathbf{1} \operatorname{div}\right)(. \mathbf{n},
$$

where $\mathbf{1}$ - a unitary tensor; $\mathbf{n}$ - normal vector external to the edge $\partial \Gamma$.

It can be easily shown that using Schwartz [2] theory and (20)-(22) the eq. (19) has the following form (see Minch \& Styś [8]):

$$
\begin{array}{r}
\mu\left\langle\left(\nabla^{2}+\frac{3 \lambda+2 \mu}{\lambda+2 \mu} \text { grad div }\right) \mathbf{u}(\mathbf{x}), \varphi\right\rangle+\langle\mathbf{b}(\mathbf{x}), \varphi\rangle= \\
\int_{\partial \Gamma}\left[\mathbf{u}(\mathbf{x}) \widetilde{\mathbf{P}}(\varphi)+(\mathbf{p}(\mathbf{x})-\widetilde{\mathbf{P}}(\mathbf{u}(\mathbf{x})) \varphi] d \partial \Gamma+\int_{\Lambda_{1} \Lambda_{2}} \mathbf{r}(\mathbf{x}) \widetilde{\mathbf{P}}(\varphi) d \Lambda\right.
\end{array}
$$

Using functional way of description with distribution in form of Dirac's- $\delta$ we can write final general differential equation of $\mathrm{RC}$ cracked plate in plane stress, appropriate 
boundary and compatibility conditions in the crack respectively:

$$
\begin{gathered}
\mu\left(\nabla^{2}+\frac{3 \lambda+2 \mu}{\lambda+2 \mu} \operatorname{grad} \operatorname{div}\right) \mathbf{u}(\mathbf{x})= \\
-\widetilde{\mathbf{P}}\left(\mathbf{r}(\mathbf{x}) \delta_{\Lambda}\right)+\left(\mathbf{p}(\mathbf{x})-\widetilde{\mathbf{P}}(\mathbf{u}(\mathbf{x})) \delta_{\partial \Gamma_{1}}+\widetilde{\mathbf{P}}\left[(\mathbf{u}(\mathbf{x})-\mathbf{u}(\mathbf{x})) \delta_{\partial \Gamma_{2}}\right],\right.
\end{gathered}
$$

where $\mathbf{b}(\mathbf{x})$ was taken in following form:

$$
\mathbf{b}(\mathbf{x})=\mathbf{P}(\mathbf{a}(\mathbf{x})) \delta_{\partial \Gamma_{2}} .
$$

\section{Ix. Model of the crack}

From the theory of defects and fundamental Green solution as a beginning support function, one can obtain discontinuity of Burgers' vector that is mathematically defined as the difference of the limits of the general displacement functions on both of the crack's edges (the crack is treated as a binary boundary), for bending state with following properties:

$$
\left[\frac{\partial w}{\partial n}\right]_{\Lambda_{1} \Lambda_{2}}(\mathbf{x})=\mathbf{r}_{2}(\mathbf{x}, \mathbf{M}), \mathbf{x} \in \Lambda_{1} \Lambda_{2},
$$

with boundary conditions $\frac{\partial r}{\partial x}\left(\Lambda_{l}\right)=\frac{\partial r}{\partial s}\left(\Lambda_{2}\right)=0$,

Here $\mathbf{r}(\mathbf{x})$ means density of defect as a continuous function for $\mathbf{x} \in \Lambda_{1} \Lambda_{2}$. The defect has been characterised by the discontinuity of the first derivative of deflection function. The assumption of internal crack $\Lambda_{1} \Lambda_{2}$ was taken. This can be easy proved. Hence on the remaining part of curve $\Lambda$ the condition (26) yields $[\partial w / \partial n]_{\Lambda}=0$, for $\mathbf{x}$ $\notin \Lambda_{1} \Lambda_{2}$. Note, that the tension continuity on the edges of the cracks is ensured by reinforcement bars which appear in the cracks.

For plane stress state of the plate as above the Burgers' vector has following form:

$$
[\mathbf{u}(\mathbf{x})]_{\Lambda_{1} \Lambda_{2}}=-\mathbf{r}_{1}(\mathbf{x}, \mathbf{N})
$$

with boundary conditions $\frac{\partial r}{\partial s}\left(\Lambda_{l}\right)=\frac{\partial r}{\partial s}\left(\Lambda_{2}\right)=0$,

Here as above $\mathbf{r}(\mathbf{x})$ means density of defect as a continuous function for $\mathbf{x} \in \Lambda_{1} \Lambda_{2}$ and $[\mathbf{u}]_{\Lambda}=0$ for $\mathbf{x}$ $\notin \Lambda_{1} \Lambda_{2}$. Equation (27) satisfies compatibility condition in the crack, where the displacement vector has a jump on a bound of crack. Because of the assumption of internal crack $\Lambda_{1} \Lambda_{2}$, hence on the remaining part of curve $\Lambda$ the condition $[\mathbf{u}]_{\Lambda}=0$ yields, for $\mathbf{x} \notin \Lambda_{1} \Lambda_{2}$. Moreover the second condition of (27) in the essential way completes the definition of the defect.

The continuity condition in the crack is written as follows:

$$
[\Re(\mathbf{u}, \dot{w})]_{\Lambda_{1} \Lambda_{2}}=0,
$$

where $\mathfrak{R}=\mathbf{n} \mathbf{C} \hat{\nabla} \quad$ (differential operator that means normal derivative to the crack line $\Lambda$ ); $\mathbf{n}$ - vector of direction cosines; $\mathbf{C}$ - elastic constants' tensor.

The properties (26)-(27) ensure discontinuity of the derivative of the deflection $w$ and the displacement vector $\mathbf{u}$ on the curve $s$ respectively (displacement of the crack for plate in plane stress and opening angle for bending slabs), whereas the property of (28) ensures continuity of tension vector $\mathbf{N}$ and bending moment $\mathbf{M}\left(\mathbf{N}=\int \sigma d h\right.$ - where $h$ means the thickness of the structure). The tension continuity on the edges of the cracks is ensured by reinforcement bars which appear in the cracks. Because of the finite number of steel bars existing in the cracks, the fulfillment of the tension conditions (28) take place only in the discrete way for the points in which reinforcement bars occur (see Fig. 2 - line $\Psi)$. Outside the reinforcement points, on the remaining edge segments of the cracks, the boundary conditions should be equal to the conditions corresponding to the free edges. Note, the nonlinear condition (28) causes conjugation of the plane stress and the bending state in an analogical way as for the solution of large deflection.

To satisfy the condition of (27), the modeling on the crack edges by the dipole normal forces for the panels with following properties was assumed:

$$
\left[u_{n}\right]_{s}=\mu^{-1} g(s),
$$

where $\mu$-Lame' constant, $g(s)$ - density of the dipole forces acting on the crack line $s$.

To satisfy the condition of (26), the modeling on the crack edges by the dipole normal moments for the slabs with following properties were assumed:

$$
\left[\varphi_{n}\right]_{s}=D^{-1} g(s),
$$

where $\varphi_{n}=$ first derivative of deflection $w_{n} ; D=$ stiffness of the cracked slab; $g(s)=$ density of the dipole normal moments acting on the crack line $s$.

Note, the dipole forces and dipole moments are selfbalanced and do not cause the changes of the loading of the construction.

\section{x. Modeling by Boundary Element Method}

The formulation based on the indirect BEM is applied. The crack is modeled using general function for displacement discontinuity and the associated fundamental solution.

The integral equations are formulated using the singular solutions associated with a point force $F_{k}$ and a point displacement discontinuity $c_{k}$. Let $(u F)_{i k}(P, S)$ represent the displacement $u_{i}$ at the field point $P$ due to a unit value of $F_{k}$ applied at the source point $S$. Similarly, let $(u r c)_{i k}(P, S)$ represent the displacement $u_{i}$ at the field point $P$ due to a unit value of $c_{k}$ applied at the source point $S$.

By distributing the point force $F_{k}$ on the boundary $\Gamma_{B}$ the displacement discontinuity $c_{k}$. on the crack boundary $\Gamma_{\Lambda}$ and bond force $\tau_{k}$ on the reinforcement line boundary $\Gamma_{\Psi}$ we obtain by superposition:

$$
\begin{gathered}
u_{i}(P)=\underset{\Gamma_{B}}{\int(u F)_{i k}(P, S) F_{k}(S) d s(S)+} \\
\int_{\Gamma_{A}}(u r c)_{i k}(P, S) c_{k}(S) d s(S)+\int_{\Gamma_{\Psi}}(u \tau)_{i k}(P, S) \tau_{k}(S) d s(S) .
\end{gathered}
$$


The boundary $\Gamma_{B}$ represents the boundaries of the body, which can be simply or multiply connected. The crack boundary $\Gamma_{\Lambda}$ represents the path of one or more cracks, which can be smooth, kinked or edge cracks. The reinforcement line boundary $\Gamma_{\psi}$ represents the line near the crack area where the bond-slip between concrete and steel bars occur.

Similarly, the stress equation can be written as:

$$
\begin{gathered}
\sigma_{i j}(P)=\int_{\Gamma_{B}}(\sigma F)_{i j k}(P, S) F_{k}(S) d s(S)+ \\
\int_{\Gamma_{A}}(\sigma r c)_{i j k}(P, S) c_{k}(S) d s(S)+\int_{\Gamma_{\psi}}(\sigma)_{i j k}(P, S) \tau_{k}(S) d s(S)
\end{gathered}
$$

where $(\sigma F)_{i j k},(\sigma \rho c)_{i j k}$ and $(\sigma \tau)_{i j k}$ are obtained by differentiation of $(u F)_{i k},(u r c)_{i k}$ and $(u \tau)_{i k}$ with respect to the field point $P$.

The curvilinear integral for the edge $\Lambda$ (31) can be interpreted as a some external forces modeling the defect by the forces taken from (29) compare with the constitutive law for crack width (9) and (27). The acting forces are selfbalanced and do not cause the increments of external loading of construction. Equation (31) is the integrodifferential equation because the right side of (31) depends directly on functions $\mathbf{u}$ and in addition functions $\mathbf{u}$ depends indirectly on the physical law (9). The rest of the equations are also the integro-differential equations.

The fundamental function $\mathfrak{\aleph}(\mathbf{x}, \mathbf{y})$ of considered problem is taken from Gefland \& Szyłow [9] solution as follows:

$$
\aleph(\rho)=\frac{1}{16 \pi} \rho^{2} \ln \left(\rho^{2}\right)
$$

where $\rho^{2}=\Phi_{1}^{2}+\Phi_{2}^{2} ; \Phi_{\alpha}=\left(x_{\alpha^{-}} y_{\alpha}\right)$ for $\alpha=1,2$.

The components of the fundamental solution $\aleph(\mathbf{x}, \mathbf{y})$ and proper derivatives of $\mathfrak{\aleph}(\mathbf{x}, \mathbf{y})$ expresses the components of general displacement vector $\mathbf{u}$ and general stress tensor $\mathbf{S}$ at the point $\mathbf{x}$, cause by unit concentrated force acting at the point $\mathbf{y}$ in the direction $x_{2}$. At the point $\mathbf{x}-\mathbf{y}=0$ the solution (33) has singularity of $1 / \mathbf{x}$ type. Hence the solution of (31) and (32) is possible in means of curvilinear integral principal value.

The final solution of singular integro-differential equations describes an accurate mathematical model of cracked RC bridge slab. The presented solution includes the discontinuity of first derivative of deflection function that is the jump on a bound of crack places and simultaneously satisfying continuity of general tension vector on both sides of the defect $\Lambda$. Such formulated model of RC cracked slab can be relatively easily written and solved in terms of boundary element method formulations. Because of fact that deformation behavior depends on the history of the loading as well as the nonlinearity of the material properties hence, the equations and definitions of the boundary element method in the rate form were assumed. According to the small strain theory, the total strain rate for inelastic problem can be divided into an elastic and inelastic part of the total strain rate tensor. Herein, the inelastic strain mean any kinds of strain field that can be considered as initial strain, i.e., plastic or viscoplastic strain rate, creep strain rate, thermal strain rate and strain rate due to other causes. So now we can write the final equations of the considered problem in terms of nonlinear BEM formulations (Brebbia et al. [10]) for fictitious traction vector $\mathbf{p}$ and body forces' $\mathbf{b}$, finally leading to the initial stresses $\sigma^{p}$ (see also Minch [11]):

$$
\mathbf{H}[\mathbf{u}, w]^{\mathrm{T}}=-\mathbf{A} \mathbf{p}+\mathbf{B} \sigma^{p}+\mathbf{F} \mathbf{x}+\mathbf{Q}(\mathbf{x}),
$$

where $\mathbf{u}$ - displacement vector; $w$ - deflection; $\mathbf{x}$ vector of unknown edge traction; $\mathbf{p}$ - vector of fictitious traction; $\sigma^{p}$ vector of initial stresses.

Here the matrices $\mathbf{H}$ and $\mathbf{A}$ are the same as for elastic analysis, matrix $\mathbf{B}$ due to the inelastic stress integral, matrix F refers to the fundamental function cause by forcing traction with vector $\mathbf{x}$, i.e., modeling density of crack opening for panel and slab respectively, while matrix of bond $\mathbf{Q}$ includes bond-slip relations and other displacements due to aggregate interlock and dowel action of reinforcement in the crack, related to displacement $\mathbf{u}$ and deflection $w$.

\section{Stress intensity factors}

When the state of stress near crack end reaches a critical value, concrete will fail by fracturing. Fracture of concrete can occur in two different ways. First as the cracking type, when the state of stress is either in the tension-tension state or tension-compression state and the stress exceeds the limit value. Second as the crushing type, when the state of stress is in the compression-compression zone and the stress exceeds the limit value. When concrete cracks, the material loses its tensile strength only normal to the crack direction, retaining its strength parallel to the crack direction.

A lot of papers deal with criteria and experimental methods for the determination of FM (fracture mechanic) parameters. Designing practice shows that fracture toughness play many times very important role. As a criterion of crack propagation of RC structures are used and applied FM parameters (see Bažant \& Cedolin [12]).

Stress intensity factors could be calculated using different methods, e.g. using the crack tip field characterization, the energy interpretation, the weight function method and line integral techniques. Many computer programs now have inbuilt FEM and BEM sub-routines which will calculate $K$ from a choice of methods. These may include direct substitutions for $K$ formula using the computed values for displacement adjacent to the crack, energy methods where $K$ is obtained by differentiating the potential or strain energy as well as evaluating the $J$ and $C$ integrals.

For presented model the method with possibilities of applying in BEM is necessary. For example first, by the $J$ integral method as used by Portela et. al. [13] in BEM where the scheme was modified slightly to incorporate the tractions on the surface of a pressurized crack. The $J$-integral method requires the evaluation of additional integral expressions for the displacement gradients. Second, by using the crack opening displacement method, based on the value of $c_{k}$ near the crack tip, as used by Sur \& Altiero [14] also in BEM. This method depends on the polynomial approximation and the location of the node. Third, by using four terms of a series solution for stresses near the crack tip, as used by Miskioglu et. al. [15] for experimental calculation of stress intensity factors, in conjunction with the least square method. A simplified representation of the series solution [15] is given as follows: 


$$
\begin{aligned}
& \sigma_{11}=\sum_{n=0}^{N} A_{n} r^{\lambda}[\cos \lambda \theta-\lambda \sin \theta \sin (\lambda-1) \theta] \\
& +\sum_{n=0}^{N} B_{n} r^{\lambda}[2 \sin \lambda \theta+\lambda \sin \theta \cos (\lambda-1) \theta] \\
& +\sum_{n=0}^{N} C_{n} r^{n}[2 \cos n \theta+n \sin \theta \sin (n-1) \theta] \\
& \sigma_{22}=\sum_{n=0}^{N} A_{n} r^{\lambda}[\cos \lambda \theta+\lambda \sin \theta \sin (\lambda-1) \theta] \\
& +\sum_{n=0}^{N} B_{n} r^{\lambda}[-\lambda \sin \theta \cos (\lambda-1) \theta] \\
& +\sum_{n=0}^{N} C_{n} r^{n}[n \sin \theta \sin (n-1) \theta] \\
& \quad \sigma_{12}=\sum_{n=0}^{N} A_{n} r^{\lambda}[-\lambda \sin \theta \cos (\lambda-1) \theta] \\
& +\sum_{n=0}^{N} B_{n} r^{\lambda}[\cos \lambda \theta-\lambda \sin \theta \sin (\lambda-1) \theta] \\
& +\sum_{n=0}^{N} C_{n} r^{n}[-\sin n \theta-n \sin \theta \cos (n-1) \theta]
\end{aligned}
$$

where $\lambda=n-\frac{1}{2}$, and

$$
A_{0}=\frac{K_{I}}{\sqrt{2 \pi}} \quad(38) ; \quad B_{0}=\frac{K_{I I}}{\sqrt{2 \pi}}
$$

The stresses in the vicinity of the crack tip (eqs.35-37) are written as a series using polar coordinates with the origin at the tip of the crack.

The least square method overcomes the shortcomings of the above two methods, however it is more sensitive then the $J$-integral to the points used in calculation.

$J$ integral may be evaluated by performing a contour $\partial \Gamma$ integration involving field variables and an integration path which encloses the crack tip as well as thermal or residual stresses arising from initial strains tensor as follows:

$$
J=\oint_{\partial \Gamma}\left(W d y-\mathbf{T} \frac{\partial \mathbf{u}}{\partial x} d s\right)+\iint_{\Gamma} \mathbf{S} \frac{\partial \mathbf{E}^{0}}{\partial x} d \Gamma
$$

where $W$ - strain energy density i.e. $W=\int \mathbf{S} d \mathbf{E} ; \mathbf{T}$ - traction forces vector; $\mathbf{E}$ - strain tensor; $\mathbf{S}$ - stress tensor.

$C^{*}$ integral is obtainable directly from $J$ integral by using the elastic analogue which states that the solution of a problem of stationary creep can be obtained from the solution of a problem of nonlinear elasticity by the following substitutions: $\mathbf{E} \Rightarrow \dot{\mathbf{E}}$ (the creep rate $d \mathbf{E} / d t$ ); $\mathbf{S} \Rightarrow$ $\dot{\mathbf{S}}$ (the effective creep stress). Thus equation (40) transforms to:

$$
C^{*}=\oint_{\partial \Gamma}\left(W^{*} d y-\mathbf{T} \frac{\partial \dot{\mathbf{u}}}{\partial x} d s\right)+\iint_{\Gamma} \dot{\mathbf{S}} \frac{\partial \dot{\mathbf{E}}^{0}}{\partial x} d \Gamma
$$

where $W^{*}$ - modified strain energy density i.e. $W^{*}=\int \dot{\mathbf{S}} d \dot{\mathbf{E}}$.

\section{Numerical calculations}

The results' correctness depends on the choice of the right type of boundary elements and a careful discretization of the structure. The influence of this on the problem to be studied cannot be neglected. The appropriate simulation of the load-carrying behavior of $\mathrm{RC}$ structure is more important than the accuracy of the numerical calculations. The question concerning what kind of numerical methods should be used cannot be answered satisfactorily.

Equation (34) must be solved numerically with iterative and incremental techniques. Iteration results are due to the fact that the right side of (34) depends directly on functions $\mathbf{u}$ and $w$. In addition functions $\mathbf{u}$ and $w$ depends indirectly on the physical law (9). The incremental computation is caused by the rate form of (34). The modified Newton-Raphson method was applied to the iteration and incremental computations.

The programme of the BEM Analysis, named PLATE, for two-dimensional problems was designed. The PLATE analysis includes the procedures of: Modelling System (MS), where the model of construction is built, the Analysis Module (AM) where the problem with iterative and incremental method is solved and finally using $M S$ the results are obtained (RES). The iterative and incremental technique used in the $(A M)$ connect all material properties with crack physical laws and edges conditions.

Let us suppose that the boundary of the slab is represented by linear elements while the part of its interior where cracks are likely to occur is discretized into a number of new boundary represented also by linear elements. The lines of broken adhesion between steel bars and concrete are the next lines of discretization. Under these assumptions, linear picewise functions are chosen to interpolate tractions, deflections and „initial stresses”, the two former over boundary elements of edges and the latter over cracks and lines of broken adhesion between steel bars and concrete. The unknowns produced by this discretization are interrelated by a series of coefficients found by integrating over boundary elements, cracks and broken adhesions' lines.

\section{Numerical example}

To show the possibilities of calculation of presented method the simply supported bridge plate $(l=10.0 \times b=5.0$ $\mathrm{m}$, thickness $\mathrm{h}=0.5 \mathrm{~m}$ ) was solved. The scheme of the plate is shown on Fig. 6.

The plate was reinforced fabric: the bottom zone with $\phi 28 \mathrm{~mm}$ bars each $15 \mathrm{~cm}$ and $\phi 12 \mathrm{~mm}$ bars each $33 \mathrm{~cm}$, the top zone with $\phi 10 \mathrm{~mm}$ bars each $33 \mathrm{~cm}$. The plate was first overloaded (to crack appear) with the concentrated force $\mathrm{P}=785 \mathrm{kN}$ acting on the top zone of the plate, then unloaded and finally loaded with uniform distributed loading $(\mathrm{p}=14$ $\mathrm{kN} / \mathrm{m}^{2}$ ).

Figure 7 shows the comparison of bending moments of elastic $\left(\mathrm{M}_{22 \mathrm{E}}\right)$ and cracked $\left(\mathrm{M}_{22 \mathrm{C}}\right)$ bridge plate. Note, that the fundamental redistribution of the bending moment $\mathrm{M}_{22}$ in the cracked zone is observed. 


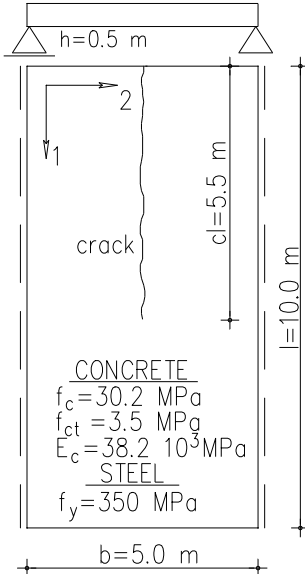

Figure 6. The scheme of the bridge slab

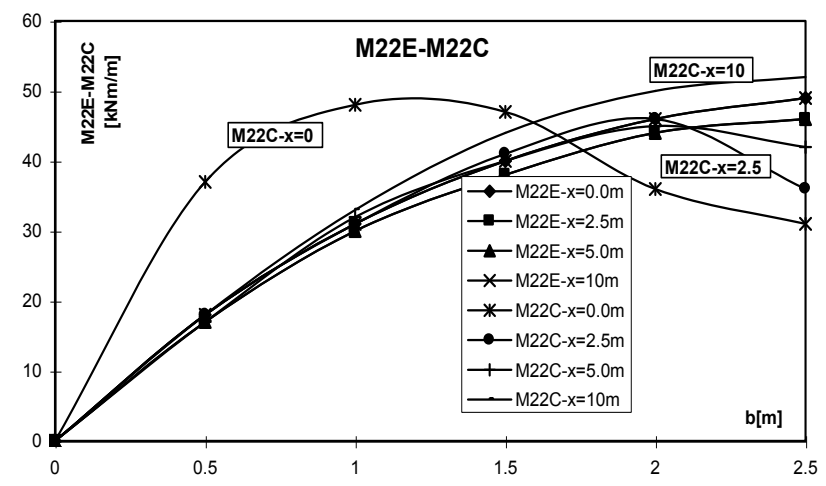

Figure 7. Comparison of elastic $\left(\mathrm{M}_{22 \mathrm{E}}\right)$ and cracked $\left(\mathrm{M}_{22 \mathrm{C}}\right)$ moments of the cracked bridge plate

\section{xIv. Conclusions and final remarks}

The boundary element method offers several advantages over finite elements such as, the possibility of working with boundary extending to infinity and having smaller systems of equations. Another very useful feature of the BEM is the admissibility of discontinuous basis function (see Brebbia et. al. [10], what give more accurate results for stress concentration regions near the cracks zones. In the finite element formulation, values of the shape functions for elements are required to be continuous along their common boundaries. On the other hand, there is no such requirement in the boundary element method. As a result, the amount of the discontinuity of the junction between elements can be used as a measure of the adequacy of the meshing. At last $\mathrm{BEM}$ require much less data than finite elements to run the same problem. This last characteristic is, without doubt, the most interesting feature of boundary elements for the practicing engineer or user.

The numerical results obtained for the problems of cracked slabs indicated that the presented methods are capable to predict sufficiently and satisfactorily response of $\mathrm{RC}$ planar structure. The considered problems are complex and very complicated. More analysis, numerical results and experimental data are still needed. More realistic descriptions of aggregate interlocking and dowel action are also necessary.

The problem of crack propagation can be solved by evaluating Rice's integral along the contour of the crack top zones, where for different specimens the stress intensity factor could be found (see Styś \& Minch [16])

\section{References}

[1] Borcz A. 1963. Podstawy teorii zarysowanych plyt żelbetowych. TNEB. Warszawa.

[2] Schwartz, L. 1966. Theory des distributions. Paris.

[3] Kupfer, H. 1973. Das Verhalten des Betons unter mehrachsiger Kurzzeitbelastung unter besonderer Berücksichtigung der zweiachsigen Beanspruchung. Deutscher Ausschuss für Stahlbeton. vol. 229. 269274.

[4] Link, J. 1976. Eine Formulierung des zweiaxialen Verformungs- und Bruchverhaltens von Beton und deren Anwendung auf die wirklichkeitsnahe Berechnung von Stahlbetonplatten. Deutscher Ausschuss für Stahlbeton. vol. 270.

[5] Dörr, K. \& Mehlhorn, G. 1979. Berechnung von Stahlbetonscheiben im Zustand II bei Annahme eines wirklichekeitsnahen Werkstoffverhaltens., Forsch. aus dem Institut für Massivbau der Technische Hochschule Darmstadt. H.39.

[6] Bažant, Z. \& Panula, L. 1978. Practical prediction on time-dependent deformations of concrete, Mater. and Struct. Res. and Testing, vol. 11(65), 307-329.

[7] Rotasy, F.S. \& Keep, B. 1982. Untersuchung der zeit- und lastabhängigen Schlupfentwicklung von einbetonierten Bewehrungsstählen, Institut für Bausoffe, Massivbau und Brandschutz, $T U$ Braunschweig.

[8] Minch, M.Y. \& Styś, D. 1996. Nonlinear analysis of RC slabs and panels by the boundary element method. International Congress of Fracture. Advances in Fracture Resistance in Materials. Tata McGrawHill Publishing Company Limited, New Delhi. vol. 1. 389-396.

[9] Gefland, I.M. \& Szyłow, G.E. 1959. Obobszczennyje funkcji i dejstwija nad nimi. Fizmatgiz. Moskva.

[10] Brebbia, C.A. \& Telles, J. \& Wróbel, L. 1984. Boundary element techniques - theory and applications in engineering. Springer Verlag, Berlin - New York

[11] Minch, M.Y. 1995. Boundary Element Analysis of RC Slabs and Panels. Proceedings of the Twenty-Fourth Midwestern Mechanics Conference. Iowa State University. Iowa USA, ISU-ERI-Ames-95002. Developments in Mechanics. vol. 18. 81-84.

[12] Bažant, Z. \& Cedolin, L. 1983. Finite element modeling of crack band propagation. Journal of Struct. Div. ASCE. Vol.109. 69-92.

[13] Portela, A. \& Aliabadi, M.H. \& Rooke, D.P. 1992. The dual boundary element method: Effective implementation for crack problems. Int. J. Num. Methods. vol.33. 1269-1287.

[14] Sur, U. \& Altiero, N.J. 1988. An alternative integral equation approach for curved and kinked cracks. Int. J. Fracture. vol.38. 25-41.

[15] Miskioglu, I. \& Mehdi-Soozani, A. \& Burger, C.P. \& Voloshin, A.S. 1987. Stress intensity factors for near edge cracks by digital image analysis. Eng. Fracture Mech. vol.27. 329-343.

[16] Styś, D. \& Minch, M.Y. 1996. Influence of mode II stress intensity factor on the fracture toughness of conrete. International Congress of Fracture. Advances in Fracture Resistance in Materials. Tata McGrawHill Publishing Company Limited, New Delhi. vol. 3. 515-522.

About Author (s):

Maciej Yan Minch
Wrocław University of Technology,
Poland
Andrzej Kmita
Wrocław University of Technology,
Poland

